\title{
Different obscurin isoforms localize to distinct sites at sarcomeres
}

\author{
Amber L. Bowman ${ }^{\mathrm{a}}$, Aikaterini Kontrogianni-Konstantopoulos ${ }^{\mathrm{a}}$, Sara S. Hirsch ${ }^{\mathrm{a}}$, \\ Sarah B. Geisler ${ }^{\mathrm{b}}$, Hugo Gonzalez-Serratos ${ }^{\mathrm{a}}$, Mark W. Russell ${ }^{\mathrm{b}}$, Robert J. Bloch ${ }^{\mathrm{a}, *}$ \\ ${ }^{a}$ Department of Physiology, University of Maryland School of Medicine, 655 W. Baltimore St, MD 21201, USA \\ ${ }^{\mathrm{b}}$ Department of Pediatrics and Communicable Diseases, University of Michigan, Ann Arbor, MI, USA
}

Received 19 February 2007; accepted 8 March 2007

Available online 15 March 2007

Edited by Michael R. Bubb

\begin{abstract}
We used four antibodies to regions of obscurin isoforms $A$ and $B$, encoded by the obscurin gene, to investigate the location of these proteins in skeletal myofibers at resting and stretched lengths. Obscurin A ( $800 \mathrm{kDa})$ which was recognized by antibodies generated to the N-terminal, Rho-GEF, and the non-modular $\mathrm{C}$-terminal domain that lacks the kinase-like domains, localizes at the level of the M-band. Obscurin B ( $900 \mathrm{kDa}$ ) which has the N-terminal, Rho-GEF, and the C-terminal kinase-like domains, localizes at the level of the $A / I$ junction. Additional isoforms, which lack one or more of these epitopes, are present at the Z-disk and $\mathrm{Z} / \mathrm{I}$ junction.
\end{abstract}

(C) 2007 Federation of European Biochemical Societies. Published by Elsevier B.V. All rights reserved.

Keywords: Obscurin; I-band; Z-disk; M-line; Stretch

\section{Introduction}

Obscurin is a recently discovered giant $(800-900 \mathrm{kDa})$ protein of striated muscle that has many Ig and several FnIII domains, as well as IQ, Src homology 3 (SH3), Rho guanine nucleotide exchange factor and pleckstrin homology (Rho$\mathrm{GEF} / \mathrm{PH}$ ) domains, and can be alternatively spliced to produce smaller products or to include kinase-like domains that may function in signaling [1-6]. In longitudinal sections of adult skeletal muscle, obscurin concentrated at the level of the Zdisks and M-bands of sarcomeres [1,3,7-9], with one report suggesting it repositioned into the I-band in stretched samples [3] but the specific obscurin isoforms present at these sites were not identified. In cross-sections of myofibers, labeling for obscurin is in a reticular pattern, indicating that obscurin surrounds the contractile apparatus [9], a position that distinguishes it from the two other giant proteins of the sarcomere, titin and nebulin. Thus, products of the obscurin gene may have unique roles in myofibrillogenesis.

Understanding the subcellular distribution of obscurin is complicated by the presence of multiple alternatively spliced forms. Obscurin is present in skeletal muscle in an $\sim 800 \mathrm{kDa}$ form, now termed obscurin A [4], characterized by a C-terminal non-modular region that binds a small form of ankyrin in the network sarcoplasmic reticulum [7,9]. An additional vari-

\footnotetext{
${ }^{*}$ Corresponding author. Fax: +1 4107068341.

E-mail address: rbloch@umaryland.edu (R.J. Bloch).
}

ant, with a larger molecular mass than obscurin A, arises from the replacement, by alternative mRNA splicing, of the nonmodular carboxy terminus with two serine/threonine kinaselike domains. Originally identified as the "giant kinase" isoform of obscurin [2,10,11] it has been more recently termed obscurin B (Fig. 1) [4]. Obscurin is also expressed as several smaller alternatively spliced variants $[1,2,4,5,12]$. Several alternatively spliced products have also been identified for obscurin's homologue in C. elegans, UNC-89 [13].

We used antibodies to different regions of obscurin, the Nterminus, the C-terminus, the Rho-GEF domain, and the Ig domain N-terminal to the first kinase-like domain (Ob68) to characterize the subcellular location of some of the alternatively spliced forms of obscurin in skeletal muscle at resting length and after stretch (Fig. 1). We localized obscurin A and obscurin $\mathrm{B}$ to the M-bands. In addition, we found obscurin B near the A/I junction, and localized distinct obscurin domains, or combinations thereof, likely to be indicative of other alternatively spliced forms, to the Z-disk and at the Z/I junction. The presence of two and possibly more different forms of obscurin at distinct sarcomeric locations suggests that these proteins play several distinct roles in organizing and stabilizing the contractile apparatus and nearby structures.

\section{Materials and methods}

\subsection{Antibodies}

We used the reverse transcriptase polymerase chain reaction (rtPCR) to amplify the coding region of the Rho-GEF domain of obscurin from rat skeletal muscle poly A+ RNA (Clontech, Palo Alto, CA) using the Superscript First Strand Synthesis System (Invitrogen, Carlsbad, CA). The primers were: reverse transcriptase, GCCACAGATCTGCTTCACCCA; forward, AGTGAATTCGTCATCCAGGAGTTGCTGAGTTC; reverse, ATCGGATCCCTAGCGCTGTGGCAGGGCAGA. This cDNA was cloned into the EcoRI/XhoI sites of pGEX-4T-1. Synthesis of the glutathione-S-transferase (GST)Rho-GEF protein was induced $(0.3 \mathrm{mM}$ IPTG, $4 \mathrm{~h})$. The protein was solubilized, purified and used to generate rabbit antibodies (Covance Research Products, Denver, PA), as described [14,15]. Antibodies were purified over affinity columns prepared with MBP-Rho-GEF. Immunodepletion experiments were performed by incubating $2 \mu \mathrm{g} / \mathrm{ml}$ of each antibody with $200 \mu \mathrm{g} / \mathrm{ml}$ of the corresponding antigen in PBS/BSA/ NGS in a total volume of $1 \mathrm{~mL}$ (PBS containing 3\% BSA, $10 \mathrm{mM}$ $\mathrm{NaN}_{3}, 5 \%$ normal goat serum: Jackson ImmunoResearch Laboratories, West Grove, PA) for $16 \mathrm{~h}$ at $4{ }^{\circ} \mathrm{C}$ and using these pre-adsorbed antibodies for immunolabeling.

We also used antibodies to: $\alpha$-actinin (mouse IgG EA53, 1:400 dilution, Sigma Chemical Co., St. Louis, MO); mouse 9D10 to the PEVK region of titin (supernatant fraction, used at 1:1 dilution, Developmental Studies Hybridoma Bank, Iowa City, IA); the N-terminal (Z-disk region) epitopes of titin (titin-Z, $3 \mu \mathrm{g} / \mathrm{ml} ;$ [12]); the N-terminal [12] and C-terminal [9] regions of obscurin A; and the Ob68-serine-threonine 

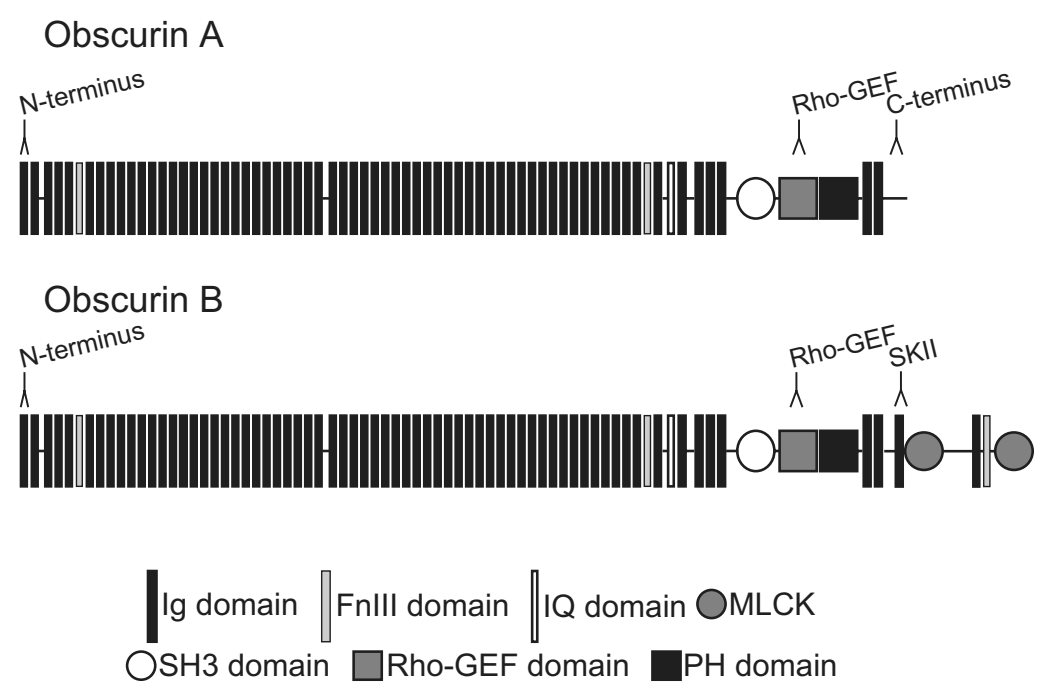

\begin{tabular}{|l|l|}
\hline Isoform & Recognized by antibodies to: \\
\hline Obscurin A & N-terminus, Rho-GEF, C-terminus \\
\hline Obscurin B & N-terminus, Rho-GEF, SKII \\
\hline
\end{tabular}

Fig. 1. Obscurin isoforms. Antibodies were prepared to distinct regions of obscurin: the N-terminal Ig domain, and the Rho-GEF domain, the nonmodular C-terminus of obscurin A and to Ob68, adjacent to the first kinase-like domain (SKII) in the giant kinase isoform, obscurin B.

kinase region of obscurin B [16]. All antibodies to titin and obscurin were generated in rabbits, except to the $\mathrm{N}$-terminal domain of obscurin and the 9D10 antibody to titin, which were created in mice.

Goat antibodies to mouse and rabbit IgGs, linked to Alexa-488 or Alexa-568 (Molecular Probes, Eugene, OR), were diluted 1:200.

\subsection{Immunoblotting}

Homogenates of adult rat muscle EDL were prepared [17] and supplemented with $50 \mathrm{U} / \mathrm{mL}$ benzonase (Novagen, Madison, WI). Aliquots $(50 \mu \mathrm{g})$ were processed for SDS-PAGE and immunoblotting [5].

\subsection{Stretched muscle}

Extensor digitorum longus (EDL) muscles, removed from adult rats with proximal and distal tendons intact, were placed in Krebs solution equilibrated with $95 \% \mathrm{O}_{2}$, and $5 \% \mathrm{CO}_{2}, \mathrm{pH}$ 7.2. Silk threads (\#4) were tied around the tendons and muscles were extended to $L_{\mathrm{o}}$, the resting tension length in vitro from the proximal to the distal myotendinous junction $[18,19]$, or to distances ranging from 1.3 to $1.7 L_{\mathrm{o}}$. (Only smaller bundles of fibers could be stretched to $1.7 L_{\mathrm{o}}$, due to the passive resistance of the connective tissue in intact muscle). The muscles were fixed immediately in 2\% paraformaldehyde in PBS for $20 \mathrm{~min}$, snap frozen, and cryosectioned [9].

\subsection{Fluorescent immunolabeling and confocal microscopy}

Frozen longitudinal sections were incubated in PBS containing 3\% BSA and 5\% non-immune goat serum (PBS/BSA/NGS) followed by primary antibodies in PBS/BSA/NGS overnight at $4{ }^{\circ} \mathrm{C}$ [9]. Samples were washed, incubated with species-specific secondary antibodies in PBS/ BSA/NGS, mounted in Vectashield, and observed with a Zeiss 410 confocal laser scanning microscope (Carl Zeiss, Inc., Tarrytown, NY).

\section{Results}

\subsection{Localization of obscurin epitopes in skeletal muscle at resting sarcomere lengths}

We used immunofluorescence to identify sites in resting muscle fibers labeled by antibodies to different epitopes of obscu- rins $\mathrm{A}$ and $\mathrm{B}$. The specificity of the $\mathrm{C}$-terminal and SKII antibodies have been previously described $[5,9,16]$. In addition, we performed immunodepletion experiments demonstrating that immunoreactivity was eliminated by preadsorbtion with the immunogen (Fig. 2A, D, G, J, insets). Antibodies to the $\mathrm{N}$-terminus primarily recognize structures at the level of the M-band, with additional but faint labeling near the Z-disk (Fig. 2A), as determined by double immunofluorescence with antibodies to epitopes of titin found at the Z-disk (Fig. 2B and $\mathrm{C}$ ). Double immunofluorescence experiments with antibodies to $\alpha$-actinin (Fig. 2E and F) indicate that the RhoGEF domain of obscurin is primarily found in structures at the level of the M-band (Fig. 2D). As previously reported, antibodies to the C-terminal region of obscurin $\mathrm{A}$ and to the Ob68-serine-threonine kinase region of obscurin B (Fig. 2G and $\mathbf{J}$ ) recognize structures at both the Z-disk and M-band, as determined by double immunofluorescence experiments with antibodies to $\alpha$-actinin (Fig. $2 \mathrm{H}, \mathrm{I}$; K, L ) $[9,16]$. These results suggest that both obscurin $\mathrm{A}$ and the epitopes within Ob68 and its adjacent kinase-like domain, associated with the B isoform, are present at the level of the M-band, though the latter may be present at lower levels. Furthermore, an isoform of obscurin or a closely related protein that contains epitopes of the non-modular C-terminal domain of obscurin $\mathrm{A}$ is likely to be present at the level of the Z-disk. However, we do not discount the possibility that Rho-GEF and N-terminal epitopes are not as accessible at the Z-disk and this is an additional site of localization of obscurin A.

\subsection{Localization of obscurin epitopes in skeletal muscle at stretched sarcomere lengths}

Epitopes of obscurin that fail to label at particular sarcomeric locations may be inaccessible. To increase accessibility, 


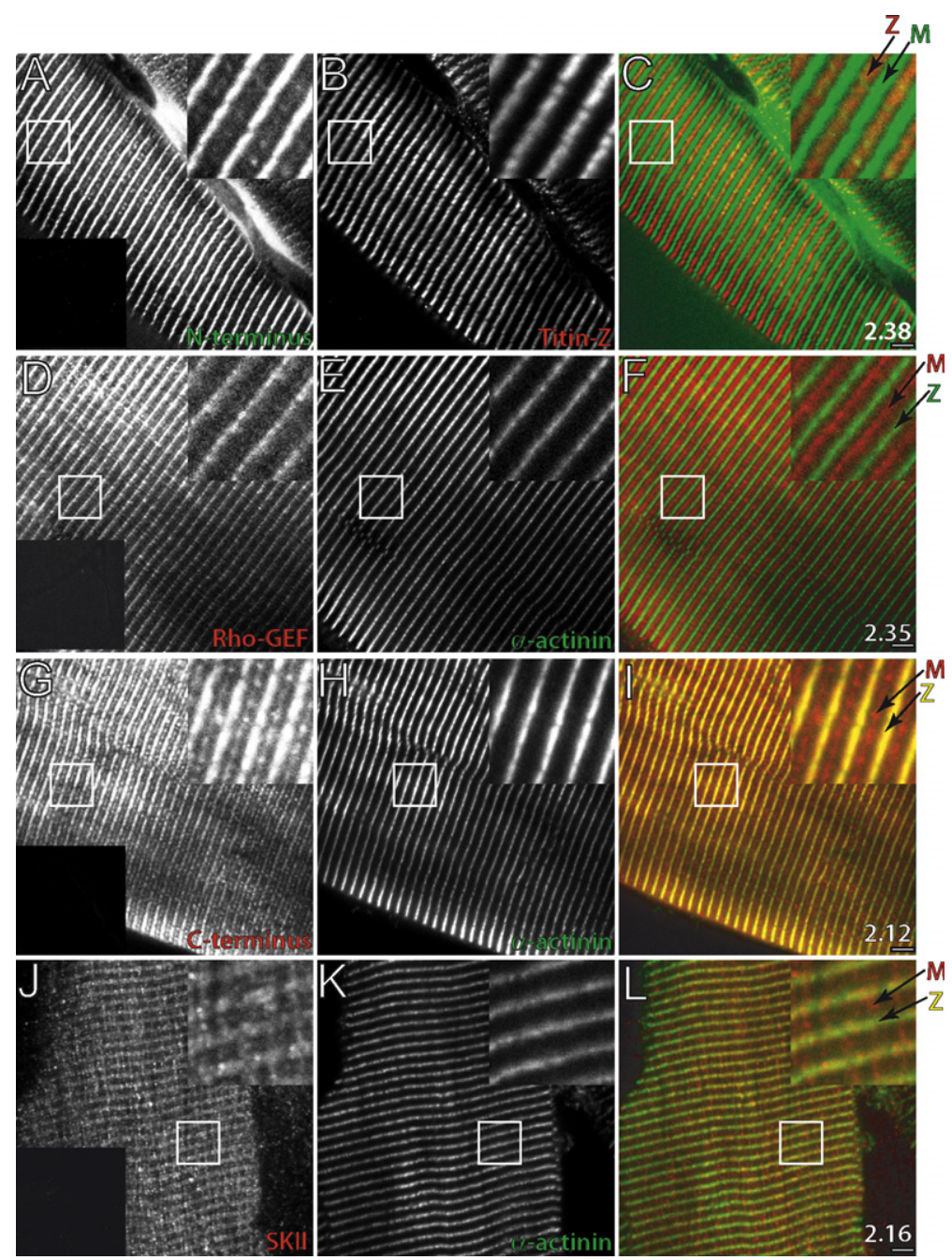

Fig. 2. Localization of obscurin isoforms in muscle at resting length. (A-F) Double immufluorescence experiments with antibodies to Z-disk epitopes of titin (B, C, red) and $\alpha$-actinin (E, F, green) to mark Z-disks, and antibodies to the N-terminus (A, C, green) and Rho-GEF domain of obscurin (D, $\mathrm{F}$, red) show that the latter primarily label at the level of the M-band. (G-L) Similarly, with the two C-terminal antibodies, to the non-modular Cterminal domain (G, I, red) and the SKII antibody to Ob68 (J, L, red). Both label primarily at the level of the Z-disk and M-band, identified by comparison with $\alpha$-actinin (H, I; K, L green). Immunodepletion experiments are shown in the lower left corners of the larger panels (A, D, G, J). Average sarcomere lengths are indicated. Bars: $5 \mu \mathrm{m}$.

we stretched EDL muscles to $130-170 \%$ of their resting length $\left(1.3-1.7 L_{\mathrm{o}}\right)$ before immunolabeling with the same anti-obscurin antibodies. Antibodies to the N-terminal and Rho-GEF domains labeled stretched samples at the level of the M-band, and also at the level of the I-band (Fig. 3A, D, G, J; compare with labeling by antibodies to Z-disk epitopes of titin, Fig. 3B, $\mathrm{E}$, and $\alpha$-actinin, Fig. $3 \mathrm{H}$ and $\mathrm{K}$ ). Labeling at the I-band was near the A/I junction, which also labeled with antibodies to the PEVK region of titin (supplemental figure 1).

Antibodies to the non-modular C-terminus labeled both the M-band and Z-disk in stretched samples, similar to unstretched muscle (Fig. 3M-O). Further increases in sarcomere length to $\sim 3.6 \mu \mathrm{m}$ did not further alter their labeling pattern, although a slight splitting could be detected at the level of the Z-disk (Fig. 3P), resulting in a decrease in overlap with $\alpha$-actinin (Fig. 3Q and R).

As in unstretched muscle, the SKII antibody that detects obscurin B labeled the M-band and the periphery of the Zdisk, near the Z/I junction, in stretched muscle. SKII antibodies also labeled structures further into the I-band in stretched muscle, as described above with antibodies to the $\mathrm{N}$-terminal and Rho-GEF domains (Fig. 3S-X).

\subsection{Relative protein levels of obscurin $A$ and $B$}

Using antibodies to the Rho-GEF domain, C-terminus and $\mathrm{N}$-terminus, we performed western blots on muscle extracts of adult rat EDL. As expected, obscurin $\mathrm{B}$ has a greater molecular mass than obscurin $\mathrm{A}$ and is recognized by antibodies to the Rho-GEF domain and the N-terminus, but not the C-terminus. In addition, obscurin $\mathrm{B}$ appears to be present at significantly lower levels (Fig. 4).

\section{Discussion}

Products of the obscurin gene play an important role in myofibrillogenesis, especially in the assembly of the M-band $[16,17,20]$, but the functions of the various, alternatively spliced gene products in this process are still unclear [1,2]. Our results suggest that the two giant forms of obscurin, termed A and B, 

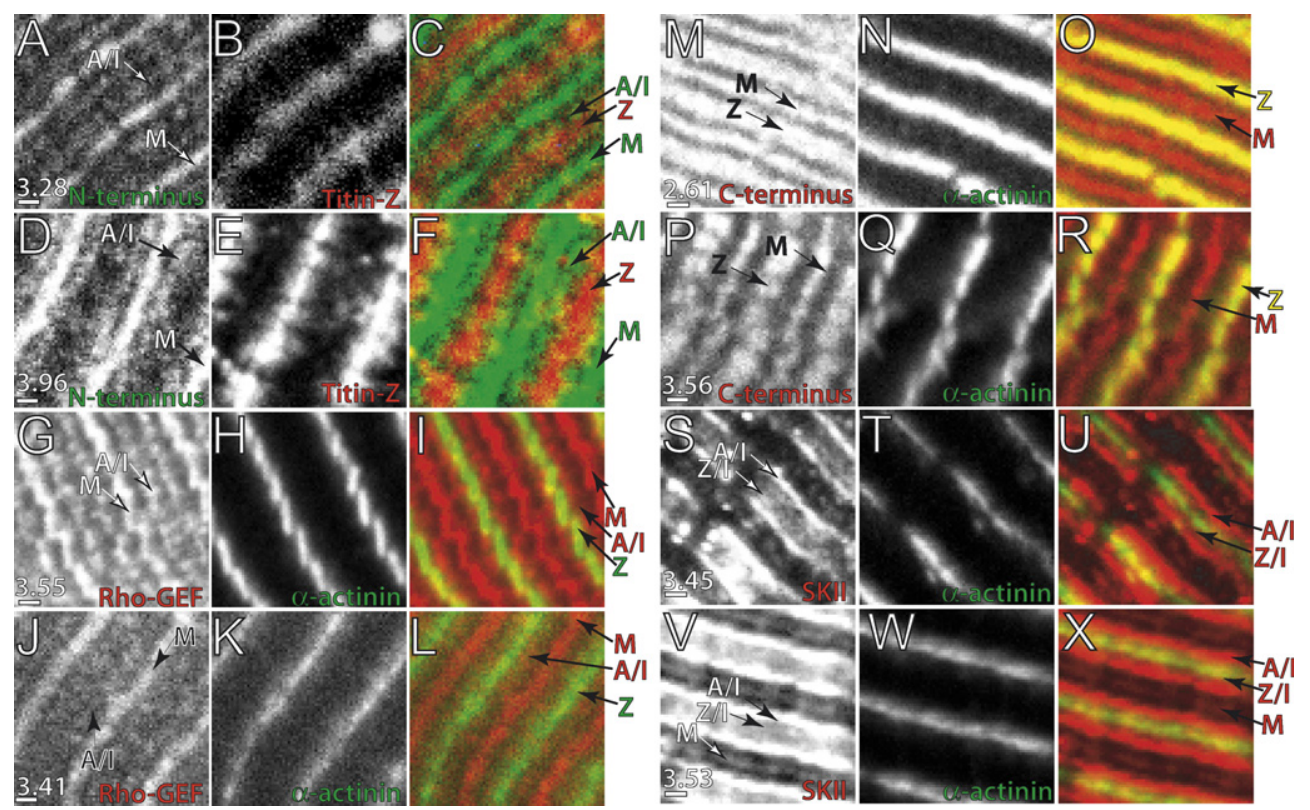

Fig. 3. Localization of obscurin isoforms in stretched muscle. As in Fig. 2, but muscle was stretched to $1.3-1.7 L_{\mathrm{o}}$ before immunolabeling. (A-F) In addition to M-bands, antibodies to the N-terminus of obscurin label the I-band near the A/I junction (A, C, D, F; green; compared to titin at the Zdisk: B, C, E, F; red). (G-L) Antibodies to the Rho-GEF domain also react with structures at both the M-band and A/I junction (G, I, J, L; red; compared to $\alpha$-actinin H, I, K, L; green). (M-R) The non-modular C-terminus of obscurin is at the level of the Z-disk and M-band (M, O; red; compared to $\alpha$-actinin: N, O; green) at $\sim 1.3 L_{\mathrm{o}}$. Greater stretching to $\sim 1.7 L_{\mathrm{o}}$ produces splitting of the C-terminal antibody labeling of the Z-disk (P, $\mathrm{R}$; red; compared to $\alpha$-actinin: Q, R; green). (S-X) Antibodies to Ob68 label at the level of the M-band and at two locations within the I-band, near the $\mathrm{A} / \mathrm{I}$ junction, and at the Z/I interface ( $\mathrm{S}, \mathrm{U}, \mathrm{V}, \mathrm{X}$; red, compared to $\alpha$-actinin: T, U, W, X; green). Average sarcomere lengths are indicated. Bars: $1 \mu \mathrm{m}$.

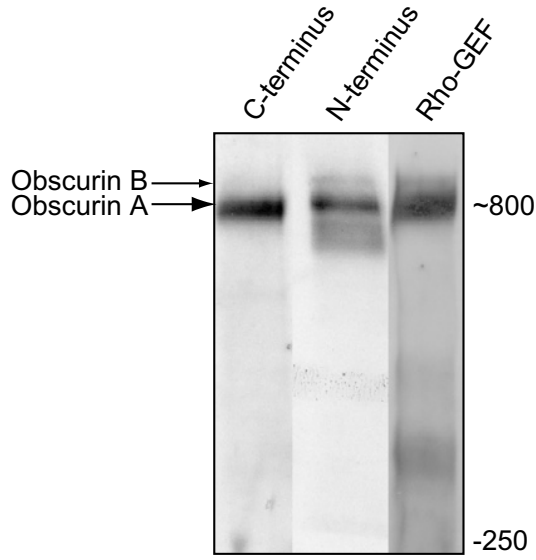

Fig. 4. Immunoblotting of obscurins A and B. In immunoblots of extracts of adult quadriceps, antibodies to the Rho-GEF domain of obscurin and the N-terminal Ig domain recognize the $800 \mathrm{kDa}$ isoform (large arrow), likely to be obscurin A, and a larger isoform that is present at much lower levels (small arrow), likely to be obscurin B. Antibodies to the C-terminus of obscurin A recognize the obscurin A isoform, but not the obscurin B isoform. Molecular masses are indicated.

are selectively enriched at different sites in skeletal muscle, and that smaller alternatively spliced forms - or post-translationally modified proteins - may be associated with the Z-disk and nearby regions of the I-band. These results are the first to document the differential distribution of different forms of obscurin in mammalian striated muscle.

We previously studied the localization of obscurin with antibodies to the non-modular C-terminus of obscurin A and deter- mined it to be at the level of Z- and M-lines in structures that surround the contractile apparatus, where it may link the sarcoplasmic reticulum to the contractile apparatus through binding to small ankyrin 1 [7,9]. We subsequently created antibodies to the N-terminal Ig, Rho-GEF, and Ob68 regions (Ob68 is adjacent to the first of two kinase-like domains of obscurin) and localized their epitopes in skeletal muscle fibers of resting and stretched lengths [3,5]. Obscurin A, as well as an epitope associated with obscurin $\mathrm{B}$, are present, at the M-band, as previously reported $[1,7,9,16]$. We find that obscurin $\mathrm{B}$ is also present at the I-band near the A/I junction. We suggest further that the isoform of obscurin present at the Z-disk that reacts with antibodies to the non-modular $\mathrm{C}$-terminus is a unique isoform which appears well after the formation of the Z-disk [11]. Our data suggest that this isoform may contain a novel N-terminus and lack the Rho-GEF domain, although we cannot now rule out the possibility that the non-modular C-terminus is more accessible to antibodies, compared to other regions of the molecule associated with the Z-disk. The SKII antibody, to domains of obscurin $\mathrm{B}$, labeled a form of the molecule in the I-band near the periphery of the Z-disk. We also observed this isoform to be recognized by antibodies to the second kinase-like domain (link 7, data not shown). This is likely to represent one of the smaller kinase-containing isoforms of obscurin, some of which are known to be expressed at low levels in skeletal muscle, but more studies of these splice forms will be needed before they can be characterized and localized in the myoplasm [2]. Whether this isoform may account for the labeling of the I-band by antibodies to Ob58/Ob59/Ob60 (formerly referred to as I48/I49/I50), as previously described by Bang et al. [3], remains to be determined. A summary of these isoforms and their sarcomeric locations is summarized in Fig. 5. 


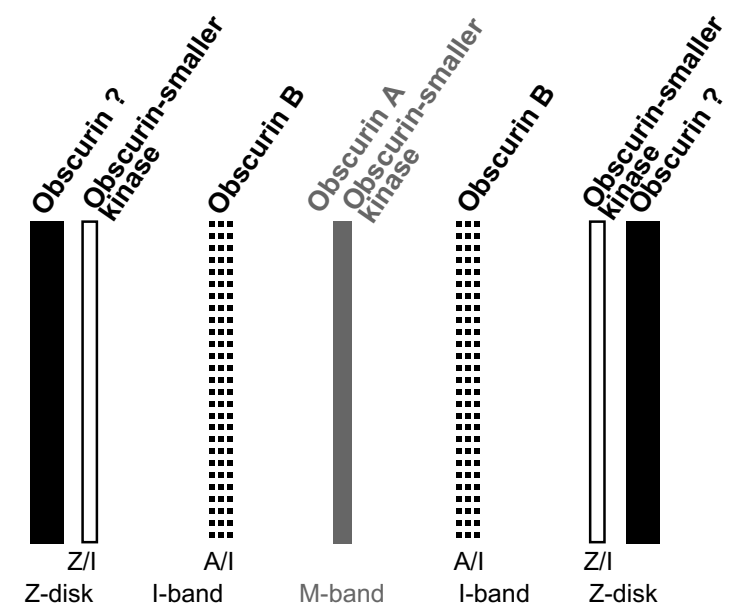

Fig. 5. Schematic representation of localization of distinct obscurin isoforms. The sarcomeric locations of each of the isoforms of obscurin we have identified are indicated. An additional isoform that has not been well characterized is likely to be located at the level of the Z-disk. See text for discussion.

The discovery of new subcellular locations for different forms of obscurin suggests that these proteins may have additional roles in myofibrillogenesis and muscle architecture that remain to be discovered, in addition to their involvement in the assembly of $\mathrm{M}$ - and A-bands or in the sarcomeric alignment of the SR $[8,11,12,16,17]$. The molecules of obscurin present at the A/I junction are near or adjacent to the PEVK region of titin, which serves as the principle elastic component of the titin filament [21]. Thus, the form of obscurin present at this site (obscurin B), may function to transduce stretch signals from this region of titin. Experiments by Borisov et al. [10] indicate that both obscurin Rho-GEF (obscurin A and B) and kinase-encoding (obscurin B) transcripts are upregulated after induction of hypertrophy, consistent with a role of obscurin isoforms $\mathrm{A}$ and $\mathrm{B}$ in these cellular processes.

Our results suggest further that other proteins or protein epitopes in the I-band are 'hidden' and thus unable to react with antibodies, and that stretching muscle may expose them, perhaps by reducing local protein densities or altering local conformations. Our findings may also indicate that some proteins originally identified as being at the Z-disk may actually associate with nearby regions of the I-band [22]. Immunofluorescence studies of stretched muscle can therefore provide a more complete characterization of the location of sarcomeric proteins.

Acknowledgements: This work was supported by Grants from the National Institutes of Health to R.J.B. (R01 HL64304), M.W.R (R01 HL075093), and A.K.K. (R01 AR52768).

\section{Appendix A. Supplementary data}

Supplementary data associated with this article can be found, in the online version, at doi:10.1016/j.febslet.2007. 03.011 .

\section{References}

[1] Young, P., Ehler, E. and Gautel, M. (2001) Obscurin, a giant sarcomeric Rho guanine nucleotide exchange factor protein involved in sarcomere assembly. J. Cell Biol. 154, 123136.

[2] Russell, M.W., Raeker, M.O., Korytkowski, K.A. and Sonneman, K.J. (2002) Identification, tissue expression and chromosomal localization of human Obscurin-MLCK, a member of the titin and Dbl families of myosin light chain kinases. Gene 282, 237-246.

[3] Bang, M.L., Centner, T., Fornoff, F., Geach, A.J., Gotthardt, M., McNabb, M., Witt, C.C., Labeit, D., Gregorio, C.C., Granzier, H. and Labeit, S. (2001) The complete gene sequence of titin, expression of an unusual approximately $700-\mathrm{kDa}$ titin isoform, and its interaction with obscurin identify a novel Z-line to I-band linking system. Circ. Res. 89, 1065-1072.

[4] Fukuzawa, A., Idowu, S. and Gautel, M. (2006) Complete human gene structure of obscurin: implications for isoform generation by differential splicing. J. Muscle Res. Cell Motil. 26, $427-434$.

[5] Kontrogianni-Konstantopoulos, A. and Bloch, R.J. (2006) Obscurin: a multitasking muscle giant. J. Muscle Res. Cell Motil. $26,419-426$.

[6] Sutter, S.B., Raeker, M.O., Borisov, A.B. and Russell, M.W. (2004) Orthologous relationship of obscurin and Unc-89: phylogeny of a novel family of tandem myosin light chain kinases. Dev. Genes Evol. 214, 352-359.

[7] Bagnato, P., Barone, V., Giacomello, E., Rossi, D. and Sorrentino, V. (2003) Binding of an ankyrin-1 isoform to obscurin suggests a molecular link between the sarcoplasmic reticulum and myofibrils in striated muscles. J. Cell Biol. 160, 245-253.

[8] Borisov, A.B., Kontrogianni-Konstantopoulos, A., Bloch, R.J., Westfall, M.V. and Russell, M.W. (2004) Dynamics of obscurin localization during differentiation and remodeling of cardiac myocytes: obscurin as an integrator of myofibrillar structure. J. Histochem. Cytochem. 52, 1117-1127.

[9] Kontrogianni-Konstantopoulos, A., Jones, E.M., Van Rossum, D.B. and Bloch, R.J. (2003) Obscurin is a ligand for small ankyrin 1 in skeletal muscle. Mol. Biol. Cell 14, 1138-1148.

[10] Borisov, A.B., Raeker, M.O., Kontrogianni-Konstantopoulos, A., Yang, K., Kurnit, D.M., Bloch, R.J. and Russell, M.W. (2003) Rapid response of cardiac obscurin gene cluster to aortic stenosis: differential activation of Rho-GEF and MLCK and involvement in hypertrophic growth. Biochem. Biophys. Res. Commun. 310, 910-918.

[11] Kontrogianni-Konstantopoulos, A., Catino, D.H., Strong, J.C. and Bloch, R.J. (2006) De novo myofibrillogenesis in C2C12 cells: evidence for the independent assembly of $\mathrm{M}$ bands and $\mathrm{Z}$ disks. Am. J. Physiol. Cell Physiol. 290, C626-C637.

[12] Kontrogianni-Konstantopoulos, A., Catino, D.H., Strong, J.C., Sutter, S., Borisov, A.B., Pumplin, D.W., Russell, M.W. and Bloch, R.J. (2006) Obscurin modulates the assembly and organization of sarcomeres and the sarcoplasmic reticulum. FASEB J. $20,2102-2111$.

[13] Small, T.M., Gernert, K.M., Flaherty, D.B., Mercer, K.B., Borodovsky, M. and Benian, G.M. (2004) Three new isoforms of Caenorhabditis elegans UNC-89 containing MLCK-like protein kinase domains. J. Mol. Biol. 342, 91-108.

[14] Frangioni, J.V. and Neel, B.G. (1993) Solubilization and purification of enzymatically active glutathione $S$-transferase (pGEX) fusion proteins. Anal. Biochem. 210, 179-187.

[15] Mercado-Pimentel, M.E., Jordan, N.C. and Aisemberg, G.O. (2002) Affinity purification of GST fusion proteins for immunohistochemical studies of gene expression. Protein Exp. Purif. 26, 260-265.

[16] Raeker, M.O., Su, F., Geisler, S.B., Borisov, A.B., KontrogianniKonstantopoulos, A., Lyons, S.E. and Russell, M.W. (2006) Obscurin is required for the lateral alignment of striated myofibrils in zebrafish. Dev. Dyn. 235, 2018-2029.

[17] Kontrogianni-Konstantopoulos, A., Catino, D.H., Strong, J.C., Randall, W.R. and Bloch, R.J. (2004) Obscurin regulates the organization of myosin into A bands. Am. J. Physiol. Cell Physiol. 287, C209-C217.

[18] Brown, L.M., Gonzalez-Serratos, H. and Huxley, A.F. (1984) Sarcomere and filament lengths in passive muscle fibres with wavy myofibrils. J. Muscle Res. Cell Motil. 5, 293-314.

[19] Brown, L.M., Gonzalez-Serratos, H. and Huxley, A.F. (1984) Structural studies of the waves in striated muscle fibres shortened 
passively below their slack length. J. Muscle Res. Cell Motil. 5, 273-292.

[20] Borisov, A.B., Sutter, S.B., Kontrogianni-Konstantopoulos, A. Bloch, R.J., Westfall, M.V. and Russell, M.W. (2006) Essential role of obscurin in cardiac myofibrillogenesis and hypertrophic response: evidence from small interfering RNA-mediated gene silencing. Histochem. Cell Biol. 125, 227-238.
[21] Linke, W.A., Ivemeyer, M., Olivieri, N., Kolmerer, B., Ruegg, J.C. and Labeit, S. (1996) Towards a molecular understanding of the elasticity of titin. J. Mol. Biol. 261, 62-71.

[22] Faulkner, G., Lanfranchi, G. and Valle, G. (2001) Telethonin and other new proteins of the Z-disc of skeletal muscle. IUBMB Life $51,275-282$. 